\title{
Strain induced fluctuations in polymer networks, melts and gels (butterfly patterns)
}

\author{
A. RAMZI, E. MENDES ${ }^{*}$, F. ZIELINSKI ${ }^{*}$, C. ROUF ${ }^{* *}$, A. HAKIKI** J. HERZ ${ }^{* *}$, R. OESER ${ }^{* * *}$, \\ F. BOUÉ and J. BASTIDE*
}

\author{
Laboratoire Léon Brillouin, CEA-CNRS, Centre d'Etudes de Saclay, 91191 Gif-sur-Yvette, France \\ * Laboratoire de Physicochimie Structurale et Macromoléculaire, CNRS-ESPCI, 10 rue Vauquelin, \\ 75251 Paris cedex, France \\ ${ }^{* *}$ Institut Charles Sadron-CRM, CNRS, Université Louis Pasteur, 6 nue Boussingault, 67083 Strasbourg \\ cedex, France \\ ${ }_{* * *}^{*}$ Institut Laue Langevin, BP. 156X, 38042 Grenoble cedex, France
}

\begin{abstract}
We present several examples of an unexpected behaviour, occurring in different types of polymer networks (permanent or temporary) containing either small or large proportions of mobile species. The spatial fluctuations of local concentration of the mobile probes appear (through neutron scattering experiments) to be anisotropically enhanced (essentially parallel to the elongation axis) when the samples are uniaxially stretched. This behaviour may arise either from an anisotropic enhancement of the thermal fluctuations under strain or from the revealing of "quenched" large scale heterogeneities. It has not been possible yet to decide which of these rather different explanations is the more satisfactory.
\end{abstract}

\section{Introduction}

Some years ago, a strong deviation with respect to the current expectations was found on studying, by small angle neutron scattering, polymer networks containing a small proportion of uncrosslinked deuterated chains [1]. Under uniaxial stretching, the intensity S(q) scattered by such systems was shown to exhibit an unexpected anisotropy: at low q, the iso-intensity lines which can be drawn when using a bidimensional detector were two lobes, one of each side of the point $\mathrm{q}=0$, having the shape of an " 8 ", or, as often quoted, of a "butterfly". Moreover the orientation of the long axis joining the centres of the lobes was parallel to the direction of stretching (see Fig. 1,2,3), along which the scattering intensity was enhanced. This orientation was called "abnormal", with respect to the classical expectations: a contribution of the network anisotropy, or any significative orientation of the deuterated uncrosslinked chains (supposed to be distributed homogeneously) would have led to a "normal" anisotropy, i:e. to ellipses with a long axis oriented perpendicular to the elongation axis and to an intensity depressed for $q$ parallel to the stretching direction. This is also the effect obtained for labelled chains chemically fixed to a network made of non-labelled chains of same species and molecular weight [2]. The phenomenon was therefore attributed to an anisotropic change of the spatial distribution of the uncrosslinked labelled chains [3], and not to their individual orientation which was proved later to be too small to be detected by SANS [4]. In other words, the concentration fluctuations of labelled chains were enhanced along the parallel direction, but not in the perpendicular one. This does not make the effect less mysterious, since the classical description (based on linear elasticity) of a stretched network containing mobile species leads to a decrease of the fluctuations along the stretching axis and to "butterflies" with a "normal" orientation (lobe axis perpendicular to the stretching) [5]. This anomaly is therefore profound and potentially fertile.

In this paper, we gather experimental facts corresponding to a much larger range of experimental situations than in the original one. We believe that these similarities are not accidental: these rather different systems have in common to exhibit a rubber-like elasticity, either permanently or temporarily (on large time scale however) and contain "probes" being free to redistribute in the sample. Then, we try to raise some questions and make some remarks, which, we hope, will be relevant to the on-going discussion of the phenomenon.

To be complete, it must be said that rather comparable "lobes" had already been observed more than twenty years ago by Inoué et al, in the case of microphase separated tri-block coploymers [6]. Moreover, some 
iso-intensity "lobes" have also been found recently, by light scattering and SANS, in the case of sheared semi-dilute solution [7-9]. Some consistency, at a certain level, might exist between all these behaviours.

\section{A few examples of "butterfly patterns" in permanent or temporary networks:}

"standard" butterfly patterns in rubbers containing some mobile labelled chains [1,4,10,11]:

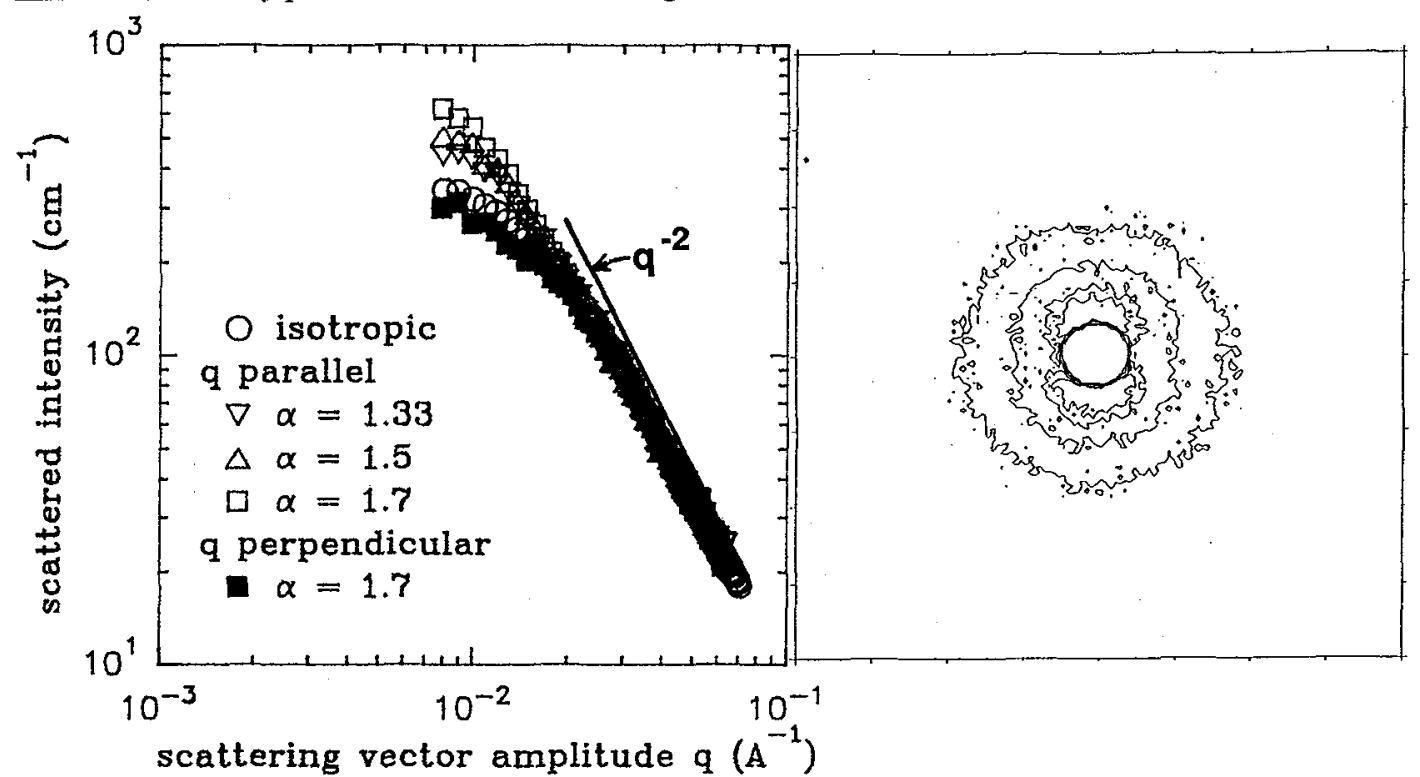

Figure 1: left: effect of the (vertical) stretching on the scattered intensity for a trifunctional PSH network with mesh of molecular weight equal to 10000 , containing $10 \%$ of free PSD chains $(M=73000)$. right: example of "butterfly" iso-intensity lines for the same sample stretched vertically by a factor $\alpha=1.7$. Experiments done at LLB Saclay.

First, we present an example of the "standard" butterfly effect, obtained in the case of a chemically crosslinked polymer network containing free deuterated chains. Although the first observation was made on Polydimethylsiloxane rubbers [1], we focus here on polystyrene networks. Repeated experiments were performed on such systems (varying the crosslinking method, the crosslinking density and the molecular weight of the free chains) and a number of the obtained results are comparable to that of the following example. In the present case, the network was prepared by end-linking of precursor chains having a relatively narrow distribution of molecular weights $\left(\mathrm{M}_{\mathrm{w}} \approx 10000, \mathrm{M}_{\mathrm{w}} / \mathrm{M}_{\mathrm{n}} \approx 1.2\right)$. The precursor chains carried hydroethyl functional groups at their ends and the crosslinking was achieved by reaction of these groups with tri-isocyanates [12]. The total polymer concentration in the reaction bath was equal to 0.24 approximately, and the polymer consisted of $90 \%$ non-deuterated di-functional chains and $10 \%$ "free" deuterated chains (i.e. carrying no functional groups), with a molecular weight $M_{w} \approx 73000\left(M_{w} / M_{n} \approx\right.$ 1.02). The obtained gels were dried under vacuum, the temperature being increased progressively up to $150^{\circ} \mathrm{C}$. The sol fraction extracted from comparable gels synthesized without free deuterated chains was shown to be ranging in between 1 and $2 \%$.

Typically a dry network sample, with dimension $7 \times 2 \times .1 \mathrm{~cm}$ is stretched by a factor $\alpha$ ranging between 1.2 and 2 in an oil bath at a temperature equal to $134^{\circ} \mathrm{C}$. At constant elongation, one observes first a decay of the stress. It then reaches an approximately constant value, assimilated with the equilibrium stress. The sample is then taken out of the bath, it becomes glassy again and can be studied easily, in this "quenched" state, by neutron scattering. On Figure 1, we show the results obtained for a "waiting time ${ }^{\text {it }} t_{R}$ of 10 minutes between elongation at $134^{\circ} \mathrm{C}$ and quenching back to room temperature. One observes, for $\mathrm{q}$ parallel to the stretching axis and at small values of the scattering vector amplitude $\mathrm{q}_{\mathrm{par}}$, a neat enhancement of the scattering intensity $S\left(q_{p a r}\right)$ as $\alpha$ is increased. On such a log-log plot, one can see that the larger the extension ratios $\alpha$, the lower the q values below which the signal signal starts to level off, at small angles. Since this saturation of the signal indicates very likely the beginning of a "Guinier regime", it means that 
the typical length scale below which some correlations are present in the system is strongly increased with the extension ratio $\alpha$. However, since here the labelled probes are chains, the relationship between this crossover and a "correlation length" is not straightforward; this question will be discused elsewhere. For q perpendicular to the stretching direction, the behaviour is entirely different: the scattered intensity $S$ (q) per is slightly depressed for $\alpha \approx 1.2$ and reaches approximately the value corresponding to the uncrosslinked system. For $\alpha>1.2, \mathrm{~S}(\mathrm{q})$ per remains approximately unchanged. The iso-intensity lines exhibit neat lobes or "butterfly" patterns", for $\alpha$ larger than 1.2 (see an example in Fig. 1). .

In the present system, butterfly patterns appear because of an increase of the spatial fluctuations, which is maximum in the direction parallel to the stretching axis and naught in the perpendicular direction. In some cases (e.g. when the crosslinking density is larger), it may happen that the scattered intensity (and therefore the spatial fluctuations) are even notably decreased in the perpendicular direction . The latter effect is also a source for the appearance of "butterflies" [4].

asymetrical polymer melts [3, 13-15]:

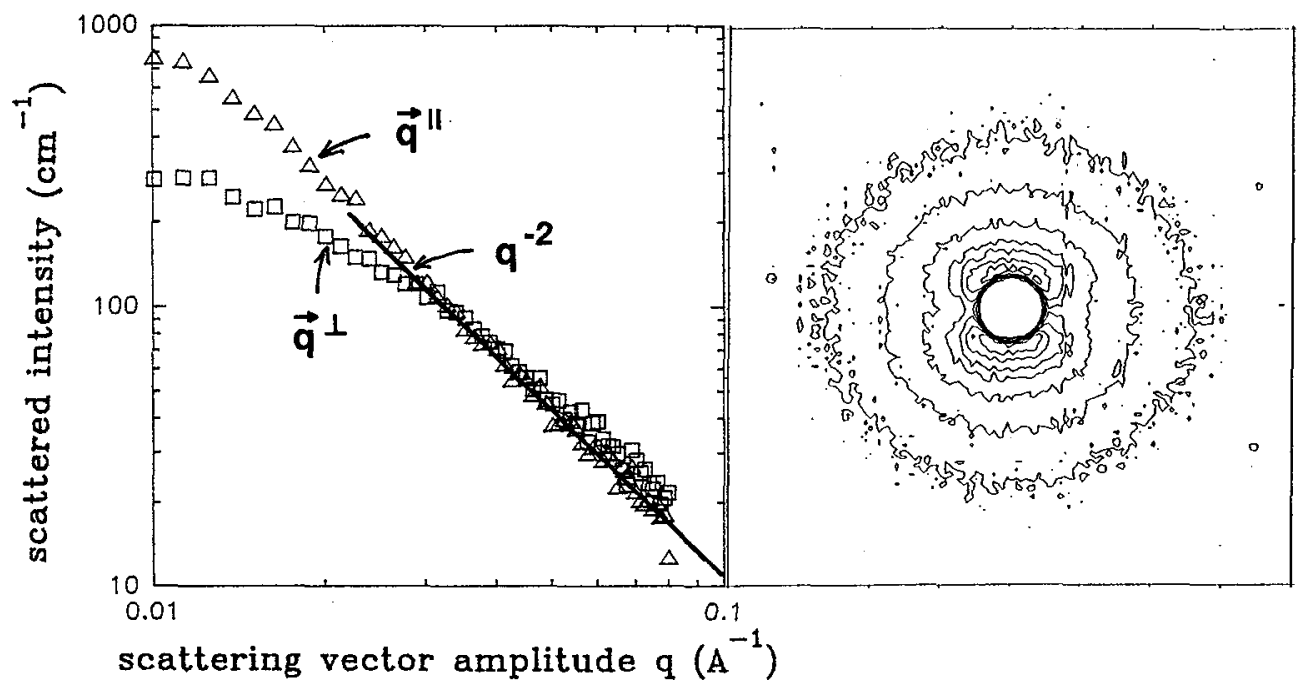

Figure 2: 1eft: Scattered intensity for an asymetrical melt (see text) elongated vertically by a factor 3 . The duration of the stress relaxation between stretching and "quenching" was $60 \mathrm{~s}$ at $140^{\circ}$ C.right: iso intensity lines for the same sample. Experiments done at LLB Saclay.

Soon after the original experiment on PDMS rubbers, it was found that "butterfly patterns" can also be observed (in a certain range of waiting times $t_{R}$ ) when the samples are not permanent networks, but only temporary ones, like polymer melts in the so-called rubbery plateau regime of relaxation [3]. In practice, one needs a "matrix" of very long (undeuterated) chains containing much shorter deuterated chains, behaving as more mobile probes. For instance, an experiment was performed on samples where the molecular weights of the matrix chains and the shorter deuterated ones (the latter representing a volume fraction of $6 \%$ ) were respectively equal to $1.110^{6}$ and 80000 . The samples were stretched by a factor $\alpha=3$ and different extents of stress relaxation (at constant elongation) before quenching the sample to room temperature were considered.

i) In the early stages of the relaxation process, i.e. for $t_{R}$ smaller than the Rouse time of the labelled chains, a classical anisotropy was observed. The iso-intensity lines were found to be ellipses having their long axis oriented perpendicular to the stretching direction. The scattered intensities extrapolated at $q=0$, for $q$ respectively parallel and perpendicular to the stretching direction, were approximately identical and equal to $\mathrm{I}(\mathrm{q} \rightarrow 0)$ for the isotropic sample (i.e. before deformation). Therefore, values of apparent radii of gyration in the directions parallel and perpendicular to the stretching axis could be extracted from the data. As expected, they were found respectively larger and smaller than the isotropic value. . 
ii) As $t_{R}$ was increased but kept of the same order of magnitude as the Rouse time of the labelled chains, the anisotropy of the elliptical iso-intensity lines decreased. At the same time, the extrapolated values $S\left(q_{p a r} \rightarrow 0\right)$ and $S\left(q_{p e r p} \rightarrow 0\right)$ remained nearly identical and equal to the isotropic value.

iii) For an even larger value of $t_{R}$, (i.e. a larger relaxation of the stress but without approaching the terminal regime of the host chains), a strong increase of the scattered intensity, for q parallel to the elongation axis, was observed, whereas, in the perpendicular direction, $S(q)$ remained close to its isotropic value. At the same time, the iso-intensity lines became butterfly-like at small angles. See the example given on Fig. 3, for which $t_{R}=60 \mathrm{~s}$ at $140^{\circ} \mathrm{C}$. In such a regime of $t_{R}$, one gets butterfly patterns which are very similar to those observed in the case of a permanent network. It must be stressed that in "chemical" networks as well, the appearance of the lobes is preceded by a regime of normal anisotropy for the lower values of $t_{R}$ [4].

\section{swollen gels under elongation [16]:}

Finally, we present an observation performed on a "statistical gel" containing a large amount of low molecular weight solvent and submitted, as before, to a uniaxial stretching. Such a gel is made from a semi-dilute solution of polystyrene $\left(M \approx 710^{7}\right)$ at a polymer volume fraction $\phi=0.1$, which is transformed into a permanent network by random reaction of tie-points between the chains (bis-chloromethyl benzene molecules with $\mathrm{SnCL}_{4}$ as a co-reagent; concentration of crosslinking molecules $c_{X}=0.8 \%$, in moles per mole of "monomer unit"). The neutron scattering contrast between the network and the solvent is achieved by swelling the gels with deuterated toluene (in the present case at saturation, $\phi \approx 0.042$, $\phi$ being the volume fraction of polymer in the gel). Therefore the mobile species are here the solvent molecules. As in the preceding cases, the scattered intensity is strongly enhanced at low q for q parallel to the stretching axis. Conversely, it is here decreased noticeably in the direction perpendicular to the stretching. See Fig. 3. As before, "butterfly patterns" are observed in the iso-intensity lines. In the present case, one can determine a correlation length $\xi$ for each extension ratio and one finds, in the parallel direction an approximate scaling law: $\xi_{\text {par }} \sim \alpha^{2}$. When the swelling of the gel is incomplete, the variation of $\xi_{\text {par }}$ with $\alpha$ is even increased $\left(\xi_{\mathrm{par}} \sim \alpha^{3}\right)$.
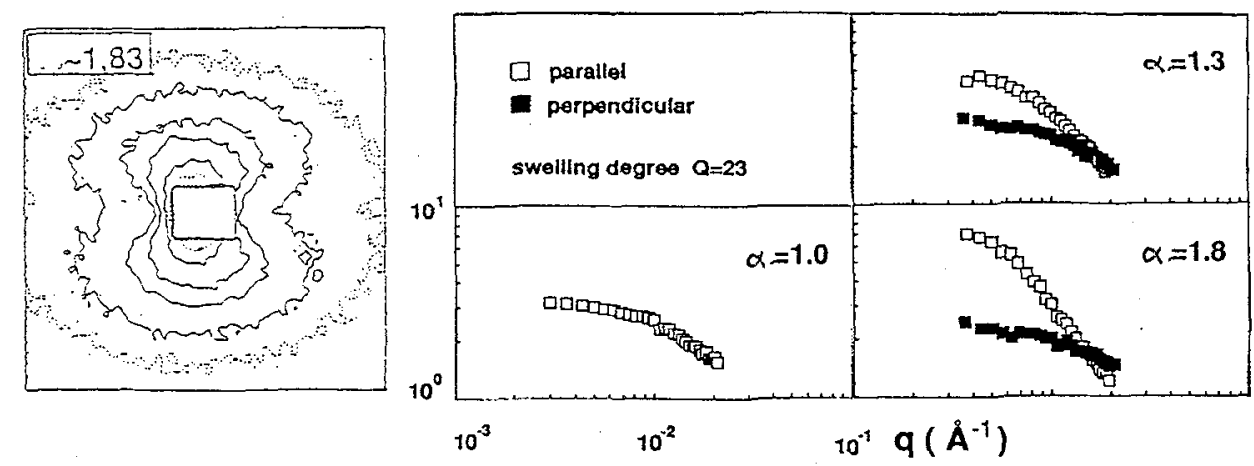

Figure 3: right: Scattered intensities (in $\mathrm{cm}^{-1}$ ) as a function of the scattering vector amplitude $\mathrm{q}$ (in $\AA^{-1}$ ), for $\mathbf{q}$ parallel and $\mathbf{q}$ perpendicular to the stretching axis, for a statistical gel, at maximum swelling in deuterated toluene $(\phi \approx 0.042)$ and different elongation ratios $\alpha$. left: several iso-intensity lines for the same gel, elongated vertically by a factor $\alpha=1.83$. Experiments done at ILL Grenoble.

\section{Thermal or quenched fluctuations?}

It is clear that the behaviours of the above binary systems, submitted to a uniaxial strain, have much in common. The phenomenon which is observed in the three cases exhibits some features which makes it similar to an approach of an anisotropic phase separation: the spatial fluctuations of one species with respect to the other one are strongly enhanced, essentially in the direction parallel to the elongation.

Several models have been proposed for this phenomenon. A first category involves a strain induced phase separation [17-19] which does not specifically lead to the patterns observed here. A second category 
considers the free energy of the deformed sample in the framework of a linear elasticity theory: under the imposed external strain, the equilibrium of forces leads, for a homogenous concentration $\phi$ of the mobile species (chains or solvent), to an affine displacement $\mathbf{u}$ of each point of the sample considered as continuous. Around these equilibrium mean values, it is further assumed that the fluctuations $\delta \phi$ and $\delta u$ are coupled differently than the mean values; this coupling assumption allows a further minimization of the free energy, corresponding to a spontaneous increase of fluctuations in the direction parallel to the stretching axis, and to a decrease in the perpendicular one [20]. This enhancement of thermal fluctuations corresponds to a sort of strain-induced anisotropic phase separation, with also an increase of the correlation length and of $S(q \rightarrow 0)$ along the elongated direction. This theory does not consider any prexistent heterogeneity of the material and is thus suitable for crosslinked as well as uncrosslinked host material (i.e. asymetrical melts or semi-dilute entangled solutions).
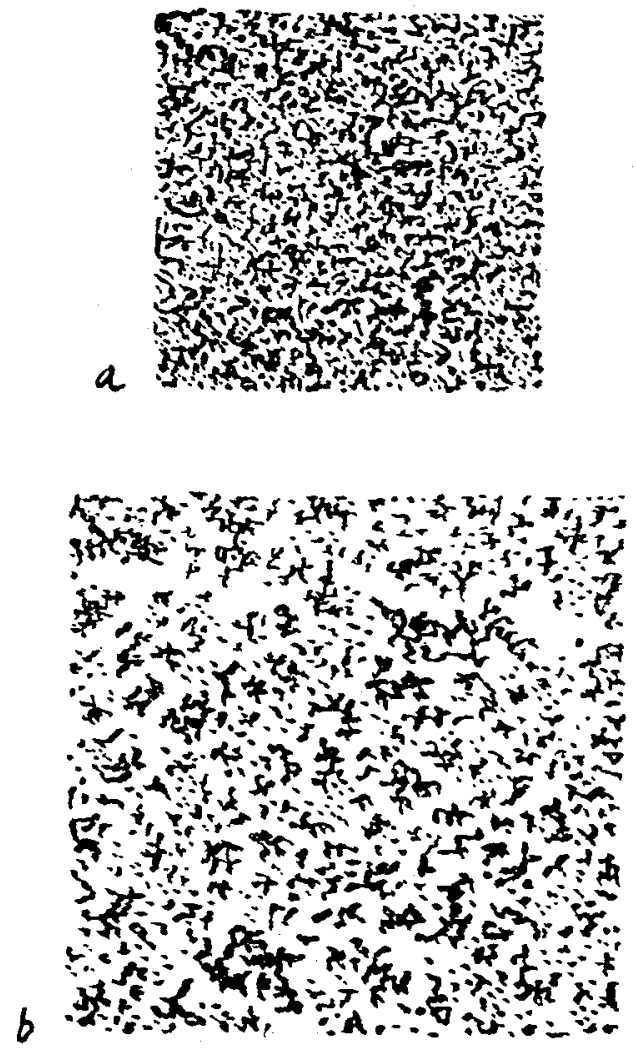

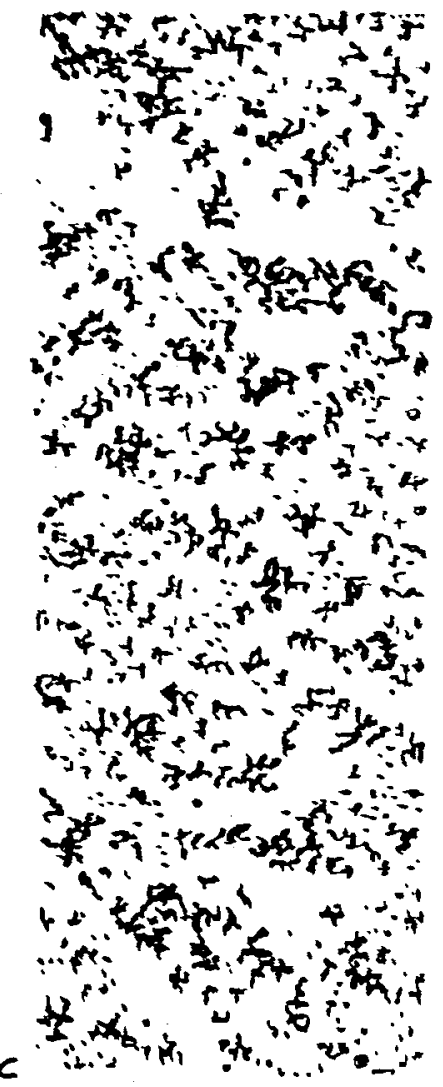

Figure 4: Schematic representation, in two dimensions of the swelling (a to $b$ ) and the (vertical) stretching ( $b$ to $c$ ) of a gel containing random inhomogeneities. The clusters represent regions where the crosslinking density is higher than on an average. They are assumed to be deformed less than the interstitial regions. For simplicity of the drawing, the clusters are kept undeformed; the distances between their centres of mass are affinely transformed. It can readily be seen that the swelling and the stretching modify strongly the spatial correlations in the system.

In a third category of models, there is no approach of a phase transition. The effect is attributed to a nonhomogeneity of structure which is assumed to preexist in the host material. Some more crosslinked or 
more entangled regions can be represented as a sort of "superstructure", which contains less of the mobile species than the network on an average. This partition achieves a "labelling" of the superstructure which may be modified by the deformation. Again, one can consider the equilibrium of forces corresponding to an homogenous concentration $\phi$ and to an affine displacement $\mathbf{u}$; this would lead first to a "classical" thermal scattering $S_{\text {therm }}(q)$, with butterfly patterns of orientation perpendicular to the one observed experimentally [22]. Then one can minimize the free energy with respect to spatial fluctuations $\delta u$ and $\delta \phi$, resulting from the presence of heterogeneities. In the reciprocal space $\langle\delta \phi(q) . \delta \phi(-q)\rangle=\mathrm{S}_{\text {froz }}(\mathrm{q})$ is considered as a additional contribution added to $S_{\text {therm }}(q)$. In other words, the spatial, frozen, fluctuations of crosslinking density $\delta v(r)$ are a perturbation and provoke a linear response $\delta \phi(q)=\chi(q) . \delta v(q)$, where the susceptibility $\chi(q)$ is $S_{\text {therm }}(q)$. In the available calculations, the crosslinking fluctuations $\delta v(r)$ are uncorrelated, and the procedure leads to a strong anisotropic increase of $S(q)$, with the same orientation as the experimental one; but the variation of the correlation length is weaker than the experimental one because it is mainly related to the one of $S_{\text {therm }}(q)$. An alternate derivation using the replica model of rubber elasticity has been given, assuming also frozen fluctuations [23].

There may be many reasons for frozen heterogeneities to be correlated on rather large length scales. For example, the crosslinking process may proceed by aggregation of elementary units. But one can find correlations even in the simple case of an ideal random crosslinking, as introduced formerly by another model [21] belonging to the third category. It was assumed that, when introducing at random some junctions between the chains of a semidilute solution, one forms an assembly of "polydisperse branched clusters" which are more crosslinked than the gel on an average. In a certain range of crosslinking density, the clusters are expected to spread over large length scales and to carry strong spatial correlations. Their internal polymer concentration may be larger than the average one, but they may stay "invisible" for the scattering, because for perfectly random formation, the intra-cluster correlations are exactly balanced by the inter-cluster ones (this is why a percolation threshold cannot be determined by scattering, except when applying a perturbation to the system). See Fig.4a. It was then argued that the swelling of such a gel could be accompanied by a sort of "unscreening" of the intra-cluster correlations (see Fig.4b) and thus lead to a strong increase of the intensity at low q: the clusters would become more and more "visible" when separated in space. Effectively, a strong enhancement of S(q) (at low q) upon swelling, consistent with this picture, was observed for "statistical" gels (the same as described above). Similarly, in the case of stretching, one may expect an "unscreening" of the intra-cluster correlations because the harder clusters should deform less than the gel on an average and then separate from each other along the direction of deformation (Fig.4c). This should lead, as observed, to an enhancement of both $S(q \rightarrow 0)$ and the correlation length $\xi$, for $q$ parallel to the elongation axis. Conversely, in perpendicular direction, the macrosccopic deformation is a contraction, similar to a deswelling, so $S(q \rightarrow 0)$ and $\xi$ should decrease. This is also observed. Putting these ingredients in a single Ornstein-Zernicke function leads to some butterfly patterns [21], with lobes aligned with the stretching axis. Because the hypotheses which are made are rather restrictive (it is assumed that the crosslinking density is such that the clusters are close to percolate), one might infer that the model is valid only in a limited range of crosslinking densities and not for all crosslinking methods. It can be argued however that "cluster-like" heterogeneities can be encountered in a much wider range of situations, as we try to show now.

\section{What is the shape of a fluctuation?}

The clusters considered above have a local crosslinking density larger than a given value $\mathrm{v} *$ (say one effective crosslink per blob). Similarly, we can "tagg" some regions of an elastic material where the local rigidity $\rho$ is larger than an arbitrary value $\rho^{*}$. Their shape will depend upon the distribution of $\rho$ and upon the choice of $\rho^{*}$. This ressembles to choosing one contour-line on the map of a mountainous area. In a certain range of altitudes, it delimitates large and branched clusters (with however a correlation length smaller than their overall size; invading such an area by water of increasing level is well known to lead to a percolation of the water areas at a certain level). Assuming that the mobile species will concentrate in the less crosslinked "valleys" of the elastic medium leads us back to the existence of cluster-like heterogeneities. The system would find advantageous, in terms of free energy, to separate the more rigid clusters (the mountains): such a way the susceptibility $\chi$ would be lower. This might hold for assymetrical polymer melts, provided the "entanglements" were randomly distributed in space. The corresponding fluctuations are indeed "frozen" on the time scale corresponding to the rubbery behaviour of melts. 


\section{Concluding remarks:}

In summary, rather different explanations can be found for the strange behaviour that polymer networks containing mobile species often exhibit under strain. On one hand, it was proposed that the spatial fluctuations which are detected are thermal and are effectively enhanced anisotropically when the system is stretched [20]. According to such a description, the phenomenon which is observed would result from a sort of strain-induced instability. Conversely, it can be imagined that the fluctuations on large length scales which are detected are, first, "quenched" (at least on time scales larger than that of the experiments) and, second, prexist to the application of a strain (they would be a kind of "blue-print" of the structural heterogeneities) [21-23]. An important point to keep in mind is that these large scale fluctuations can remain undetectable for a scattering experiment in a state where their distribution in space is totally random, but can be "revealed", through an "unscreening" process, by the application of a strain (e.g. give rise to "butterflies" under stretching).

But it is difficult to know, from static scattering data, which of these two types of mechanisms is the more relevant. We hope that, in the future, dynamical scattering experiments will allow to decide. Another option is to try to observe experimentally some predictions made by the models. Following the way of the "strain induced instabilities", one expects that, for a large enough elongation of a sufficiently crosslinked network, a real (but anisotropic) microphase separation could be attained. This is why we have performed the following experiment and found, again, a surprising behaviour and not the predicted one.

\section{An anomaly in the anomaly:}

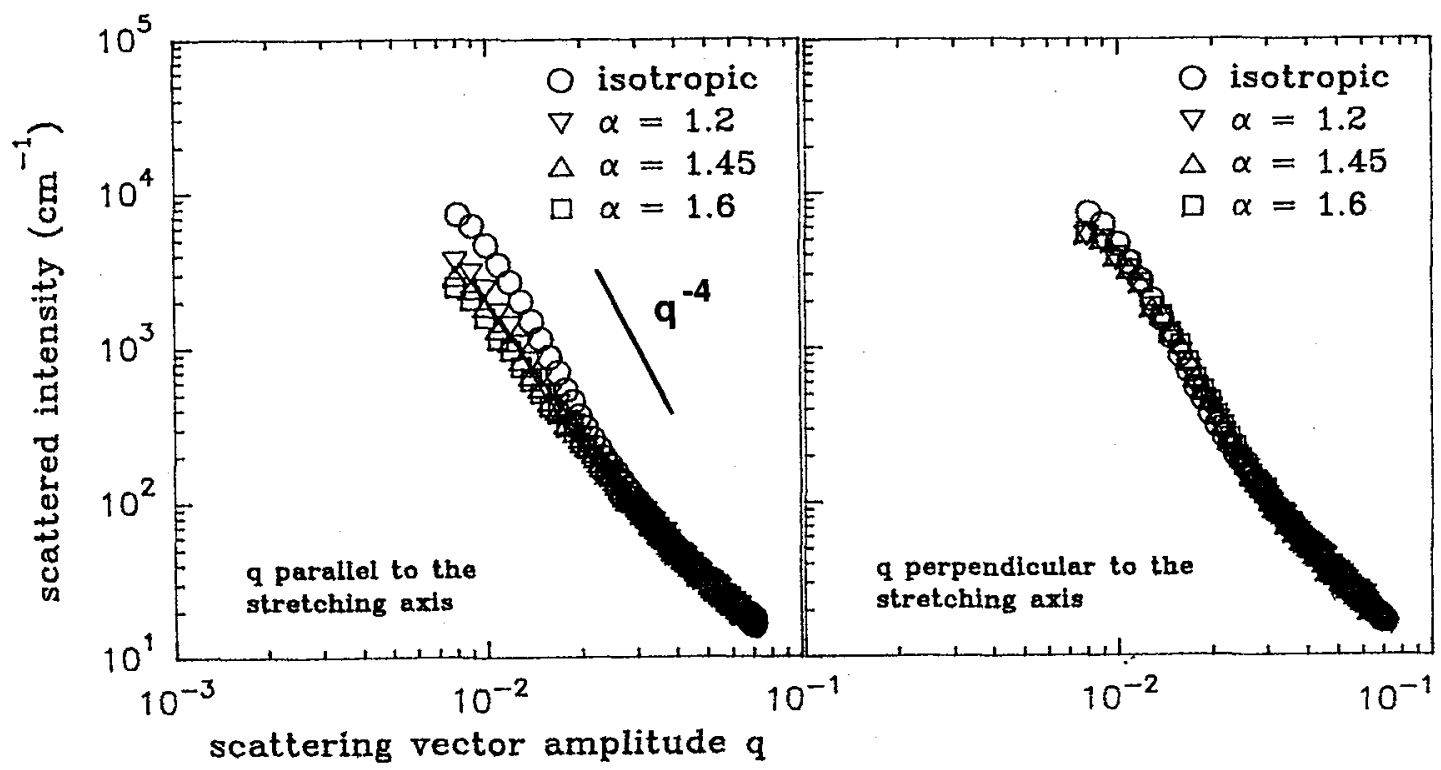

Figure 5: Effect of the stretching on the scattered intensity for a trifunctional PSH network with a "mesh" of molecular weight equal to 5000 , containing $10 \%$ of free PSD chains $(M=73000)$. $\alpha$ denotes the stretching ratio; the duration of the relaxation between stretching and quenching the sample back to room temperature was $10 \mathrm{mn}$ at $134^{\circ} \mathrm{C}$.

In the case of the "standard "butterflies" presented above the molecular weight of the elementary chains of the nework was equal to 10000 and that of the free labelled chains was equal to 73000 . Then, we synthesized samples using the same crosslinking method (end-linking through trifunctional junctions), but with precursor chains two times shorter $(M=5000)$. The labelled chains were kept the same and were put in the same proportions: 10\%). In the isotropic state, the signal was found to be very different than in the preceding case at intermediate $\mathrm{q}$ values. As shown on Figure 5, the signals almost follows a $\mathrm{q}^{-4}$ 
dependence in between presumably a Guinier regime (the beginning of a saturation at low q's) and a crossover towards $\mathrm{q}^{-2}$ at large $\mathrm{q}$ 's. This behaviour indicates very likely that a sort of microphase separation occured between the network and the free chains. A more surprising fact, however, is that the signal is now lowered in the parallel direction, as the sample is stretched. In the perpendicular direction, one observes only a slight decrease of the intensity at low q.. As a consequence, the iso-intensity lines recover then a nearly elliptical shape with a long axis perpendicular to the stretching direction. For this more strongly crosslinked system, the butterfly patterns therefore disappear.

Here, the stretching seems to induce a kind of "remixing" of the separated free chains (in the parallel direction essentially) Such a behaviour could be related to the well known fact that gels swell under stretching, which can be interpreted through the classical theories of swelling [24]. It is too early to conclude, but note that such an explanation would disagree with the picture of strain induced instabilities.

\section{References:}

1. R. Oeser, C. Picot, J. Herz, in "Polymer Motions in Dense Systems", D. Richter and T. Springer Eds., p.104, Springer Proceedings in Physics 29 (1988)

2. see e.g. F. Boué et al, Prog. Col. Pol. Sci.75 152 (1987)

3. J. Bastide, M. Buzier, F. Boué p. 112, Springer Proceedings in Physics 29 (1988); and F. Boué, J. Bastide, M. Buzier, Springer Proceedings in Physics 42 A. Baumgärtner, C. Picot, Eds.(1989)

4. F. Zielinski, M. Buzier, C. Lartigue, J. Bastide, F. Boué, Prog. Coll. Polym.Sci. 90115 (1992) and F. Zielinski, Thesis, Université Pierre et Marie Curie, Paris VI (1991) available on demand (contact

F. Boué)

5. A. Onuki, in "Space-Time Organization in Macromolecular fluids", p. 54 , F. Tanaka, T. Ohta and M. Doi Eds., Springer Proceedings in Physics (1989)

6. T. Inoue, M. Moritani, T. Hashimoto, H. Kaway, Macromolecules, 4500 (1971)

7. X. L. Wu, D.J. Pine, P. K. Dixon, Phys. Rev. Lett. 662408 (1991)

8.T. Hashimoto, T. Kume, J. Phys. Soc. Jap. 611839 (1992)

9. P. Lindner, F. Boué, submitted (1993)

10. A. Ramzi, F. Zielinski, A. Hakiki, M. Buzier, J. Bastide, F. Boué. in "Proceedings of ACS-PMSE, "Neutron Scattering from Polymers" 204th National Meeting" Washington DC August 1992

11. J. Bastide, F. Boué, E. Mendes, F. Zielinski, M. Buzier, G. Beinert, R. Oeser, C. Lartigue

"Networks 91; Dusek and Kuchanov Eds., VSP The Netherlands, p. 119-145 (1992),

12. A. Hakiki, J. G. Zilliox, G. Beinert, J. Herz, Polymer, 332796 (1992)

13. J. L. Barea, R. Muller, C. Picot, in "Polymer Motions in Dense Systems", D. Richter and T. Springer Eds., p.86, Springer Proceedings in Physics 29 (1988)

14. F. Boué, J. Bastide, M. Buzier, A. Lapp, J. Herz, T. Vilgis, J. Polym. Colloid Sci., 269195 (1991) 15. J.W. van Egmond, D.E. Werner, G. Fuller, J. Chem. Phys. 967742 (1992)

16. E. Mendes, P. Lindner, M. Buzier, F. Boué, J. Bastide, Phys. Rev. Lett. 661595 (1991) and

J. Bastide, E. Mendes, F. Boué, M. Buzier, P. Lindner, Makromol. Chem., Macromol. Symp, 4081 (1990)

17. F. Brochard, P. G. de Gennes, C. R. Acad. Sci. Ser. 2, 306699 (1988)

18. R.G. Larson, Rheol. Acta 31497 (1992)

19. P. Olmsted, S. Milner, preprint

20.Y. Rabin, R. Bruinsma, Eur. Phys. Lett. 2079 (1992)

21.J. Bastide, L. Leibler, Macromolecules 212647 (1988), J. Bastide, L. Leibler, J. Prost, Macromolecules 231821 (1990)

22. A. Onuki, J. Phys. II, France 245 (1992)

23. S. Panyukov, JETP Lett. (1992)

24. K. Dusek, W. Prins, Adv. Polym. Sci. 6, 1 (1969) 